\title{
SPECTRAL MAPPING THEOREMS AND PERTURBATION THEOREMS FOR BROWDER'S ESSENTIAL SPECTRUM
}

\author{
BY
}

\author{
ROGER D. NUSSBAUM
}

\begin{abstract}
If $T$ is a closed, densely defined linear operator in a Banach space, F. E. Browder has defined the essential spectrum of $T$, ess $(T)$ [1]. We derive below spectral mapping theorems and perturbation theorems for Browder's essential spectrum. If $T$ is a bounded linear operator and $f$ is a function analytic on a neighborhood of the spectrum of $T$, we prove that $f(\operatorname{ess}(T))=\operatorname{ess}(f(T))$. If $T$ is a closed, densely defined linear operator with nonempty resolvent set and $f$ is a polynomial, the same theorem holds. For a closed, densely defined linear operator $T$ and a bounded linear operator $B$ which commutes with $T$, we prove that ess $(T+B) \subseteq$ ess $(T)$ + ess $(B)=\{\mu+\nu: \mu \in \operatorname{ess}(T), \nu \in$ ess $(B)\}$. By making additional assumptions, we obtain an analogous theorem for $B$ unbounded.
\end{abstract}

Introduction. Let $T$ be a closed, densely defined linear operator on a Banach space $X$. F. E. Browder [1] defined the essential spectrum of $T$, ess $(T)$, to be the set of $\lambda \in \sigma(T)$, the spectrum of $T$, such that at least one of the following conditions holds: (1) $R(\lambda-T)$, the range of $\lambda-T$, is not closed; (2) $\lambda$ is a limit point of $\sigma(T)$; (3) $\bigcup_{r>0} N\left[(\lambda-T)^{r}\right]$ is infinite dimensional, where $N(A)$ denotes the null space of a linear operator $A$. Browder proved that $\lambda_{0} \notin$ ess $(T)$ if and only if $(\lambda-T)^{-1}$ is defined for $0<\left|\lambda-\lambda_{0}\right|<\delta$ and the Laurent expansion of $(\lambda-T)^{-1}$ around $\lambda_{0}$ has only a finite number of nonzero coefficients with negative indices.

Recall that a closed, densely defined operator $T$ is called a Fredholm operator if and only if $\operatorname{dim} N(T)<\infty, R(T)$ is closed, and codim $R(T)<\infty$. Let us write $G=\{\lambda \in C \mid \lambda-T$ is a Fredholm operator $\}$. Since $G$ is known to be an open set let us denote by $G_{i}, i \in I$, the open connected components of $G$. Gohberg and Kreĭn [2] proved (though they never formally defined the essential spectrum) that $\lambda_{0} \notin$ ess $(T)$ if and only if $\lambda_{0}$ belongs to an open connected component $G_{i}$ which has a nonempty intersection with the resolvent set of $T$.

There are several other characterizations of ess $(T)$. D. Lay [5] has shown that if $C$ is a compact, bounded linear operator such that $C: D(T) \rightarrow D(T)$ and $(C T)(x)=(T C)(x)$ for $x \in D\left(T^{2}\right)$, then ess $(T+C)=$ ess $(T)$ and that conversely ess $(T)$ is the largest subset of $\sigma(T)$ which remains invariant under such perturbations. Other authors have described the essential spectrum of $T$ in terms of the ascent, descent, nullity and defect of $T$, but we shall not need these results.

Received by the editors July 7, 1969.

AMS Subject Classifications. Primary 4710, 4730, 4748.

Key Words and Phrases. Browder's essential spectrum, spectral mapping theorems, perturbation theorems, noncompact perturbations. 
In this paper we shall find Lay's result especially useful. In the first section we shall establish spectral mapping theorems. If $B$ is a bounded linear operator and $f$ is a function analytic on a neighborhood of $\sigma(B)$, we shall prove that ess $(f(B))$ $=f(\operatorname{ess}(B))$. If $T$ is a closed, densely defined linear operator with nonempty resolvent and $p$ is a polynomial, we shall show that ess $(p(T))=p(\operatorname{ess}(T))$.

In the second section we consider perturbation theorems for the essential spectrum. Let $T$ be a closed, densely defined linear operator and $B$ a bounded linear operator such that $B: D(T) \rightarrow D(T)$ and $(B T)(x)=(T B) x$ for $x \in D(T)$. Then we shall prove that ess $(T+B) \subset \operatorname{ess}(T)+\operatorname{ess}(B)=\{\nu+\mu \mid \nu \in \operatorname{ess}(T), \mu \in \operatorname{ess}(B)\}$. If $0 \notin \sigma(B)$ or if $0 \notin$ ess $(B)$ and $\sigma(T) \neq C$ (either hypothesis implies that $B T$ is closed), then we shall show that ess $(B T) \subset \operatorname{ess}(B)$ ess $(T)=\{\mu \nu \mid \mu \in \operatorname{ess}(B), \nu \in \operatorname{ess}(T)\}$. Finally, we shall establish a perturbation theorem which makes no boundedness assumption on $B$. Let $T$ and $B$ be closed, densely defined linear operators with $D(T) \subset D(B)$. Let $D=\{x \in D(T) \mid T x \in D(B)\}$ and assume that $B: D \rightarrow D(T)$ and $(T B) x=(B T) x$ for $x \in D$. Assume that $T+B$ is closed, $\rho(T+B)$ is nonempty, and ess $(T)$ is compact. Then there is a compact subset $S$ of ess $(B)$ such that ess $(T+B) \subset$ ess $(T)+S=\{\nu+s \mid \nu \in$ ess $(T), s \in S\}$.

I would like to thank Richard Beals for a number of helpful discussions about the last mentioned theorem. In particular he suggested a simplified proof of an early version of the theorem, and it is that proof which will be given here.

1. Spectral mapping theorems for the essential spectrum. We begin with some trivialities. It is clear from the definition of ess $(B)$ that if $X$ is a finite dimensional vector space and $B: X \rightarrow X$ is a linear operator, then ess $(B)$ is empty. Thus we shall always assume that $X$ is an infinite dimensional Banach space, since our theorems are trivial otherwise. In this case, if $B: X \rightarrow X$ is a bounded linear operator, ess (B) is nonempty, since otherwise $\lambda-B$ would be Fredholm of index zero for all $\lambda \in C$, whence by Theorem 3.2 of [2], $X$ would be finite dimensional.

THEOREM 1. Let $B$ be a bounded linear operator of a Banach space $X$ into itself. Let $f$ be an analytic function defined on an open neighborhood of $\sigma(B)$. Then ess $(f(B))=f($ ess $(B))$.

Proof. Let $G$ be the open neighborhood of $\sigma(B)$ on which $f$ is defined and let $\Gamma$ be a Jordan curve (not necessarily connected) contained in $G$ and enclosing $\sigma(B)$. We can select $\varepsilon_{0}>0$ such that $\Gamma$ encloses $N_{\varepsilon_{0}}(\sigma(B))=\left\{\lambda \mid d(\lambda, \sigma(B))<\varepsilon_{0}\right\}$.

First, let us show that ess $(f(B)) \subset f($ ess $(B))$. To prove this it suffices to show that ess $(f(B)) \subset f\left(N_{\varepsilon}(\right.$ ess $\left.B)\right)$ for all $\varepsilon, \varepsilon_{0} \geqq \varepsilon>0$. For suppose $\mu_{0} \in f\left(N_{\varepsilon}\right.$ (ess $\left.\left.B\right)\right)$ for all $\varepsilon, \varepsilon_{0} \geqq \varepsilon>0$. Then we can find a sequence of points $\nu_{n}$ such that $d\left(\nu_{n}\right.$, ess $\left.B\right)$ $\rightarrow 0$ and $f\left(\nu_{n}\right)=\mu_{0}$. Since ess $B$ is compact, we can find a convergent subsequence $\nu_{n_{i}} \rightarrow \nu_{0} \in \operatorname{ess}(B)$, and $f\left(\nu_{0}\right)=\mu_{0}$.

Thus, select $\varepsilon, \varepsilon_{0} \geqq \varepsilon>0$. Since $\sigma(B)-N_{\varepsilon}$ (ess $\left.B\right)$ is a compact set consisting of isolated points, let $\sigma(B)-N_{\varepsilon}$ (ess $\left.B\right)=\left\{\nu_{1}, \ldots, \nu_{m}\right\}$. Let $C_{1}, \ldots, C_{m}$ be disjoint circles 
about $\nu_{1}, \ldots, \nu_{m}$ and such that the only point of $\sigma(B)$ enclosed in $C_{i}$ is $\nu_{i}$. Write $P=(1 / 2 \pi i) \sum_{j=1}^{m} \int_{C_{j}}(\nu-B)^{-1} d \nu$ and recall that $P$ is a finite dimensional projection commuting with $B$ (see, for instance, [2] or [4]). Since we can assume ess $(B)$ nonempty, let us select $\nu_{0} \in \operatorname{ess}(B)$ and set $B_{1}=B-B P+\nu_{0} P$. We claim that $\sigma\left(B_{1}\right) \subset N_{\varepsilon}$ (ess $\left.B\right)$. To see this, set $M=(I-P)(X)$ and note that $B_{1}|M=B| M(B$ clearly maps $M$ into itself). By the functional calculus for bounded linear operators, it is known that $\mu-B \mid M$ is one-to-one and onto $M$ for $\mu$ inside one of the $C_{i}$, and since the only other points in $\sigma(B)$ lie in $N_{\varepsilon}$ (ess $\left.B\right), \mu-B_{1} \mid M$ is one-to-one and onto $M$ for $\mu \notin N_{\varepsilon}$ (ess $\left.B\right)$. On the other hand, if we write $V=P X$,

$$
\mu-B_{1}\left|V=\left(\mu-\nu_{0}\right)\right| V,
$$

so $\mu-B_{1}$ is one-to-one and onto $V$ for $\mu \neq \nu_{0}$. It follows that $\mu-B_{1}$ is one-to-one and onto $X$ for $\mu \notin N_{\varepsilon}$ (ess $B$ ).

Our next claim is that ess $(f(B))=\operatorname{ess}\left(f\left(B_{1}\right)\right)$. (Notice that $f\left(B_{1}\right)$ is defined since $\sigma\left(B_{1}\right) \subset N_{\varepsilon}$ (ess $\left.B\right) \subset G$, and in fact $\Gamma$ encloses $\sigma\left(B_{1}\right)$.) Our claim will follow from Lay's theorem if we can show that $K=f\left(B_{1}\right)-f(B)$ is compact and commutes with $f(B)$. Commutativity is clear. To see compactness notice that for $\mu \in \Gamma$ (so $\mu \in \rho(B) \cap \rho\left(B_{1}\right)$ ), $\mu-B$ and $\mu-B_{1}$ take $M$ into $M$ and $V$ into $V$. Since $X=$ $M \oplus V$, it follows that $(\mu-B)^{-1}$ and $\left(\mu-B_{1}\right)^{-1}$ take $M$ into $M$ and $(\mu-B)^{-1} x$ $=\left(\mu-B_{1}\right)^{-1} x$ for $x \in M$. Thus, for $x \in X,\left(\mu-B_{1}\right)^{-1} x-(\mu-B)^{-1} x=\left(\mu-B_{1}\right)^{-1}(P x)$ $-\left(\mu-B_{1}\right)^{-1}(P x)$, and we see finally that

$$
f\left(B_{1}\right) x-f(B) x=\frac{1}{2 \pi i} \int_{\Gamma} f(\mu)(\mu-B)^{-1} x d \mu=\left(f\left(B_{1}\right)-f(B)\right)(P x),
$$

a finite dimensional operator.

With the above observation we can complete the first half of the proof. We have ess $(f(B))=\operatorname{ess}\left(f\left(B_{1}\right)\right) \subset \sigma\left(f\left(B_{1}\right)\right)$. But by the ordinary spectral mapping theorem, $\sigma\left(f\left(B_{1}\right)\right)=f\left(\sigma\left(B_{1}\right)\right) \subset f\left(N_{\varepsilon}(\right.$ ess $\left.B)\right)$.

Conversely, we want to show that ess $(f(B)) \supset f($ ess $B)$. Assume not, so that there exists $\mu_{0} \in f($ ess $B)$-ess $f(B)$. Since $\mu_{0} \notin \operatorname{ess} f(B)$, take a circle $C$ about $\mu_{0}$ which contains no other points of $\sigma(f(B))$. Let $P_{1}=(1 / 2 \pi i) \int_{C}(\mu-f(B))^{-1} d \mu$, a finite dimensional projection, and let $M_{1}=\left(I-P_{1}\right) X$. It follows as above that for $\nu \in \Gamma,(\nu-B)^{-1}: M_{1} \rightarrow M_{1}$ and thus $f(B) \mid M_{1}=f\left(B \mid M_{1}\right)$. Also as above, we know that $\mu_{0}-f(B) \mid M_{1}$ is a one-to-one map of $M_{1}$ onto $M_{1}$. Thus if $f\left(\nu_{0}\right)=\mu_{0}$ for $\nu_{0} \in \operatorname{ess}(B), \nu_{0} \notin \sigma\left(B \mid M_{1}\right)$, since if it were, we would have

$$
\mu_{0} \in \sigma\left(f\left(B \mid M_{1}\right)\right)=\sigma\left(f(B) \mid M_{1}\right) .
$$

Now we can show that $\nu_{0} \notin$ ess $(B)$, which gives a contradiction. We use Browder's original definition. If we set $V_{1}=P_{1} X$, the range of $\nu_{0}-B$ is $M_{1} \oplus\left(\nu_{0}-B\right) V_{1}$, the span of a closed subspace and a finite dimensional subspace, hence closed. Since $V_{1}$ is finite dimensional $(\nu-B) \mid V_{1}$ must be one-to-one and onto $V_{1}$ for 
$0<\left|\nu-\nu_{0}\right|<\delta$, and since $\nu_{0}-B \mid M_{1}$ is one-to-one and onto $M_{1}, \nu-B \mid M_{1}$ is one-toone and onto $M_{1}$ for $\left|\nu-\nu_{0}\right|<r, r>0$. Thus $\nu-B$ is one-to-one and onto for $0<\left|\nu-\nu_{0}\right|<\min (r, \delta)$ and $\nu_{0}$ is an isolated point of $\sigma(B)$. Finally, we clearly have $\operatorname{dim}\left(\bigcup_{j>0} N\left(\nu_{0}-B\right)^{j}\right) \leqq \operatorname{dim} V_{1}$. Q.E.D.

REMARK 1. Theorem 1 was proved by T. T. West [8] for the case that ess $(B)$ $=\{0\}$ (the so-called "Riesz operators") and $f(0)=0$.

If we try to generalize Theorem 1 to unbounded linear operators $T$, we have the problem of defining $f(T)$. If $f$ is a polynomial, however, there is basically no problem and we can obtain an analogue of Theorem 1. First we need a lemma. This result is not difficult; a proof is given by Goldberg [3, pp. 107-108].

LEMMA 1 (GoldBerg). Let $T$ be a closed, densely defined linear operator such that $\sigma(T) \neq C$. Let $p$ be a polynomial of degree $n$; then $p(T)$, with domain $D\left(T^{n}\right)$, is a closed, densely defined linear operator and $\sigma(p(T))=p(\sigma(T))$.

LeMma 2. Let $T$ be a closed, densely defined linear operator on $X$ such that $\sigma(T) \neq C$. Let $F$ be a bounded, finite dimensional linear operator such that $(\nu-T)^{-1} F$ $=F(\nu-T)^{-1}$ for $\nu \in \rho(T)$. Then (a) $F(X) \subset \bigcap_{j>0} D\left(T^{j}\right)$; (b) $(F T) x=(T F) x$ for $x \in D(T)$; (c) for all $j \geqq 1,(F+T)^{j} x-T^{j} x=E_{j} x$ for $x \in D\left(T^{j}\right), E_{j}$ a bounded, finite dimensional linear operator such that $E_{j}: D\left(T^{j}\right) \rightarrow D\left(T^{j}\right)$ and $T^{j} E_{j} x=E_{j} T^{j} x$ for $x \in D\left(T^{j}\right) ;$ (d) for any polynomial $p$, ess $(p(T+F))=\operatorname{ess}(p(T))$.

Proof. Select $\nu \in \rho(T)$. Since $D(T)=(\nu-T)^{-1} X$ and since $(\nu-T)^{-1} F=F(\nu-T)^{-1}$, $F: D(T) \rightarrow D(T)$. Showing $(F T) x=(T F) x$ for $x \in D(T)$ is equivalent to showing that $F(\nu-T) x=(\nu-T) F x$ for $x \in D(T)$, and to prove this we simply apply $(\nu-T)^{-1}$ to both sides.

If we set $V=F(X)$, by assumption, $V$ is finite dimensional. Since $D(T)$ is dense in $X$ and $F$ is bounded, $F(D(T))$ is a dense subspace of $V$ and hence $F(D(T))=V$, so that $V \subset D(T)$. Because $(F T) x=(T F) x$ for $x \in D(T), T: V \rightarrow V$ and thus $\nu-T: V \rightarrow V$. However, $V$ is finite dimensional, so we have $(\nu-T)^{-1}: V \rightarrow V$ and in fact $V=(\nu-T)^{-1} V$. It follows that $V=(\nu-T)^{-j} V$ for all $j \geqq 1$, so $V \subset D\left(T^{j}\right)$ for all $j \geqq 1$.

To prove (c), note that because $(T F) x=(F T) x$ for $x \in D(T)$, we can write $(T+F)^{j} x-T^{j} x=A_{j} F x$ for $x \in D\left(T^{j}\right), A_{j}$ a linear operator defined on $D\left(T^{j}\right)$. However, by our above work, $V \subset D\left(T^{j}\right)$ and since $V$ is finite dimensional, $\left\|A_{j} v\right\| \leqq M\|v\|$ for $v \in V$. Thus we see that for $x \in X,\left\|A_{j} F x\right\| \leqq M\|F x\| \leqq M\|F\|\|x\|$, so that $E_{j}=A_{j} F$ is a bounded linear operator. By using the commutativity of $F$ and $T$, we see that for $v \in D\left(T^{j}\right)$,

$$
A_{j} v=\sum_{i=0}^{j-1}\left(\begin{array}{l}
j \\
i
\end{array}\right) T^{i} F^{j-i-1} v
$$

and since $F x \in D\left(T^{2 j}\right)$ for any $x$, it follows immediately that $A_{j} F x \in D\left(T^{j}\right)$ for any $x$. Finally, since $T^{k} F x=F T^{k} x$ for $x \in D\left(T^{k}\right)$ (by repeatedly using $(F T) x=(T F) x$ for $x \in D(T)$ ), it is easy to see that $T^{j} E_{j} x=E_{j} T^{j} x$ for $x \in D\left(T^{j}\right)$. 
By using (c), we see that $p(T+F) x-p(T) x=E x$ for $x \in D\left(T^{n}\right)(n=$ degree of $p)$, $E$ a finite dimensional, bounded linear operator such that $E: D\left(T^{n}\right) \rightarrow D\left(T^{n}\right)$ and $p(T) E x=E p(T) x$ for $x \in D\left(T^{n}\right)$. It follows by Lay's theorem that

$$
\text { ess }(p(T+F))=\operatorname{ess}(p(T)) \text {. }
$$

THEOREM 2. Let $T$ be a closed, densely defined linear operator on a Banach space $X$ and assume that $\sigma(T) \neq C$. Then if $p$ is a polynomial, $p(T)$ is closed and ess $(p(T))$ $=p($ ess $(T))$.

Proof. The proof follows the basic outlines of Theorem 1, so we shall be sketchy.

Let $V_{R}(0)$ denote the closed ball of radius $R$ about 0 in $C$ and let $V_{R}^{\prime}(0)$ denote the complement of $V_{R}(0)$. We want to show that ess $(p(T)) \subset p($ ess $T)$ and in order to prove this it suffices to prove that

$$
\text { ess }(p(T)) \subset p\left(N_{\varepsilon}(\text { ess } T) \cup V_{R}^{\prime}(0)\right) \text { for all } \varepsilon>0, R>0 \text {. }
$$

For suppose we have established the above inclusion and $\lambda_{0} \in \operatorname{ess}(p(T))$. Unless $p$ is constant, in which case the theorem is trivial, there exists an $R$ so large that $p\left(V_{R}^{\prime}(0)\right) \subset V_{\left|\lambda_{0}\right|}^{\prime}(0)$, so that $\lambda_{0} \in p\left(N_{\varepsilon}(\right.$ ess $\left.T) \cap V_{R}(0)\right)$. If we select $\varepsilon_{n} \rightarrow 0$ and $\mu_{n} \in N_{\varepsilon_{n}}$ (ess $\left.T\right) \cap V_{R}(0)$ such that $p\left(\mu_{n}\right)=\lambda_{0}$, then since $\mu_{n} \in V_{R}(0)$, a compact set, we can assume $\mu_{n} \rightarrow \mu \in$ ess $(T)$ and $\lambda_{0}=p(\mu)$.

Consider

$$
\begin{aligned}
& \left(\sigma(T)-N_{\varepsilon}(\text { ess } T)\right) \cap V_{R}(0) \\
& \quad=\left\{\mu_{1}, \ldots, \mu_{m}\right\}\left(\sigma(T)-N_{\varepsilon}(\text { ess } T)=\left\{\lambda \mid \lambda \in \sigma(T), \lambda \notin N_{\varepsilon}(\text { ess } T)\right\}\right) .
\end{aligned}
$$

If we let $C_{1}, \ldots, C_{m}$ be disjoint circles such that $\mu_{j}$ is the only point of $\sigma(T)$ contained in $C_{j}$, and if we set $P=(1 / 2 \pi i) \sum_{j=1}^{m} \int_{C_{j}}(\lambda-T)^{-1} d \lambda$, then just as before $P$ is a finite dimensional projection and $P: X \rightarrow D(T)$. If we select $\mu_{0}$ with $\left|\mu_{0}\right|>R$ and if we set $F=-T P+\mu_{0} P$, the same proof as before shows that $\sigma(T+F)$ $\subset N_{\varepsilon}($ ess $T) \cup V_{R}^{\prime}(0)$. Since $P$ is finite dimensional, $F$ is finite dimensional; and since $P$ is continuous as a map from $X$ to $D(T)$ with the graph topology and $T$ is continuous from $D(T)$ with the graph topology to $X, F$ is bounded. It is also clear that for $\nu \in \rho(T),(\nu-T)^{-1} F=F(\nu-T)^{-1}$. It follows by Lemma 2 that

$$
\text { ess }(p(T))=\operatorname{ess}(p(T+F)) \subset \sigma(p(T+F))=p(\sigma(T+F)) \subset p\left(N_{\varepsilon}(\operatorname{ess} T) \cup V_{R}^{\prime}(0)\right) \text {. }
$$

The proof that $p(\operatorname{ess} T) \subset$ ess $(p(T))$ is essentially the same as in Theorem 1, and we omit it. We just note that a little more care must be exercised since $T$ is not everywhere defined. Q.E.D.

2. Perturbation theorems for the essential spectrum. To begin this section let us establish some notation. We shall say that linear operators $T$ and $B$ satisfy hypothesis one $\left(H_{1}\right)$ if $T$ is a closed densely defined linear operator with domain $D(T), B$ is a bounded linear operator such that $B: D(T) \rightarrow D(T)$, and $(T B)(x)$ $=(B T)(x)$ for $x \in D\left(T^{2}\right)$. Condition $H_{1}$ is Lay's definition of " $T$ commutes with 
$B$ " [5]. We shall say that linear operators $T$ and $B$ satisfy $H_{2}$ if $T$ and $B$ are the same as above, but we strengthen the commutativity condition to $(T B)(x)=(B T)(x)$ for $x \in D(T)$.

Our next lemma is concerned with the behavior of $\sigma(T+B)$ when $T$ and $B$ satisfy $H_{1}$. In the case that $T$ is bounded, this lemma is well known and follows by Banach algebra techniques. In the general case the lemma has been proved by J. T. Marti [6].

Lemma 3 (MARTI). Let $T$ and $B$ be linear operators satisfying $H_{1}$. Then $T+B$ is closed and $\sigma(T+B) \subset \sigma(T)+\sigma(B)=\{\mu+\nu \mid \mu \in \sigma(B), \nu \in \sigma(T)\}$. If $\sigma(T)$ is empty, we interpret $\sigma(T)+\sigma(B)$ as the empty set.

The following lemma is also proved by Marti [6]. If $T$ is bounded, it follows by Banach algebra techniques without the assumption that $0 \notin \sigma(B)$.

LemMa 4 (MARTI). Let $T$ and $B$ be linear operators satisfying $H_{2}$. Assume that $0 \notin \sigma(B)$. Then $B T$ is a closed linear operator, and

$$
\sigma(B T) \subset \sigma(B) \sigma(T)=\{\mu \nu \mid \mu \in \sigma(B), \nu \in \sigma(T)\} .
$$

If $\sigma(T)$ is empty, $\sigma(B) \sigma(T)$ is interpreted as empty.

RemARK 2. Marti actually proves Lemma 3 under the assumption that $B: D(T) \rightarrow D(T)$ and $(T B) x=(B T) x$ for $x \in D(T)$. However, if $\sigma(T)=C$, Lemma 3 is immediate. If $\sigma(T) \neq C$ and $\nu \in \rho(T), H_{1}$ implies that $B(\nu-T)^{-1} x=(\nu-T)^{-1} B x$ for $x \in D(T)$ (apply $(\nu-T)$ to both sides), whence by continuity $B(\nu-T)^{-1} x$ $=(\nu-T)^{-1} B x$ for all $x$. If $u \in D(T), u=(\nu-T)^{-1} x$, so that

$$
(\nu-T) B u=(\nu-T) B(\nu-T)^{-1} x=B x=B(\nu-T) u,
$$

and Marti's commutativity condition holds.

Remark 3. Marti considers closed, densely defined linear operators $T$ and $S$ such that $D(T) \subset D(S), S: D(T) \rightarrow D(T)$, and $(T S) x=(S T) x$ for all $x \in D(T)$ such that $T x \in D(S)$. This generality is illusory, however. For suppose $\rho(T) \neq \varnothing$ and select $\nu \in \rho(T)$. For $x \in D(S),(\nu-T) S(\nu-T)^{-1} x=S x$, by the commutativity condition. If we let $Y$ denote the Banach space $D(T)$ with the graph topology $\left(\|x\|_{Y}=\|x\|+\|T x\|\right)$, it is easy to see that $S$ is a closed linear operator as a map from $Y$ to $Y$. Thus $S$ is bounded as a map from $Y$ to $Y$. Since $(\nu-T)^{-1}: X \rightarrow Y$ is bounded and $(\nu-T): Y \rightarrow X$ is bounded, $\left\|(\nu-T) S(\nu-T)^{-1} x\right\| \leqq M\|x\|$. It follows that $\|S x\| \leqq M\|x\|$ for $x \in D(S)$, and since $S$ was assumed closed, $D(S)=X$ and $S$ is bounded.

The same remark applies to Lay's article [5].

THEOREM 3. Let $T$ and $B$ be linear operators satisfying $H_{1}$. Then $T+B$ is closed and ess $(T+B) \subset \operatorname{ess}(T)+\operatorname{ess}(B)=\{\nu+\mu \mid \nu \in \operatorname{ess}(T), \mu \in \operatorname{ess}(B)\}$. If ess $(T)$ is empty, this means that ess $(T+B)$ is empty. 
Proof. If $\sigma(T)=C$, ess $(T)=C$ and we are done. Thus we assume $\sigma(T) \neq C$. Now we establish some simple commutativity relationships. By Remark 2 we know that $(T B) x=(B T) x$ for $x \in D(T)$. By the proof of Remark 2 we have $(\nu-T)^{-1} B=B(\nu-T)^{-1}$ for $\nu \in \rho(T)$, and it follows (by applying $\mu-B$ to both sides of the equation) that $(\mu-B)^{-1}(\nu-T)^{-1}=(\nu-T)^{-1}(\mu-B)^{-1}$ for $\mu \in \rho(B)$, $\nu \in \rho(T)$. The latter equation implies that $(\mu-B)^{-1}$ takes $D(T)$ into $D(T)$, so that we see (by applying $\mu-B$ to both sides of the equation) that $(\mu-B)^{-1} T x$ $=T(\mu-B)^{-1} x$ for $x \in D(T)$.

Select $\lambda_{0} \in \operatorname{ess}(T+B)$. We want to show that $\lambda_{0} \in \operatorname{ess}(T)+\operatorname{ess}(B)$. Suppose we can show that for every $\varepsilon>0, \lambda_{0} \in N_{\varepsilon}($ ess $T)+N_{\varepsilon}($ ess $B)$. This will imply that for every $\varepsilon>0, \lambda_{0} \in N_{2 \varepsilon}$ (ess $T+$ ess $B$ ). Since ess $T$ is closed and ess $B$ is compact, ess $T+$ ess $B$ is closed, and it will follow that $\lambda_{0} \in \operatorname{ess} T+\operatorname{ess} B$.

Thus take $\lambda_{0} \in$ ess $(B+T)$ and select $\varepsilon>0$. Let $R$ be a constant such that $\left|\lambda_{0}\right|<R$ and $|\mu|<R$ for $\mu \in N_{\varepsilon}(\sigma(B))$. As in $\S 1$, let $V_{2 R}(0)=\{x \mid\|x\| \leqq 2 R\}$. Since spectral points which are not in the essential spectrum are isolated, we know that $\sigma(T)$ $\cap\left(V_{2 R}(0)-N_{\varepsilon}(\operatorname{ess} T)\right)=\left\{\nu_{1}, \ldots, \nu_{n}\right\}$ and $\sigma(B)-N_{\varepsilon}(\operatorname{ess} B)=\left\{\mu_{1}, \ldots, \mu_{m}\right\}$. Let $D_{1}$, $\ldots, D_{n}$ be disjoint circles about $\nu_{1}, \ldots, \nu_{n}$, each $D_{\text {, containing no points of } \sigma(T)}$ except $\nu_{j}$. Similarly, let $C_{1}, \ldots, C_{m}$ be disjoint circles about $\mu_{1}, \ldots, \mu_{m}$ such that each $C_{j}$ contains no point of $\sigma(B)$ except $\mu_{j}$. As in $\S 1$ we know that

$$
Q=\sum_{j=1}^{m} \frac{1}{2 \pi i} \int_{C_{j}}(\mu-B)^{-1} d \mu
$$

is a bounded, finite dimensional projection; similarly

$$
P=\sum_{j=1}^{n} \frac{1}{2 \pi i} \int_{D_{j}}(\nu-T)^{-1} d \nu
$$

is a bounded, finite dimensional projection, and in fact $P: X \rightarrow D(T)$ is continuous from $X$ to $D(T)$ with the graph topology.

We are now in a position to complete the proof. Take $\nu_{0},\left|\nu_{0}\right|>2 R$, and select $\mu_{0} \in$ ess $(B)$ (since we always assume $X$ infinite dimensional, ess $(B) \neq \varnothing$ ). Consider the bounded, finite dimensional linear operator $F=\nu_{0} P-T P+\mu_{0} Q-B Q$. By using the commutativity relations of the first paragraph of the proof, it is easy to see that for $\nu \in \rho(T),(\nu-T)^{-1} F=F(\nu-T)^{-1}$. It follows by Lemma 2 that $F(X)$ $\subset \bigcap_{j \geq 1} D\left(T^{j}\right)$ and $T F x=F T x$ for $x \in D(T)$. It is also clear that $F B=B F$, so that $(T+B) F x=F(T+B) x$ for $x \in D(T+B)=D(T)$. It follows by Lay's theorem that ess $(T+B+F)=$ ess $(T+B)$.

The next step is to make use of the form of $F$. We have

$$
\text { ess }(B+T+F) \subset \sigma\left(\left(B+\mu_{0} Q-B Q\right)+\left(T+\nu_{0} P-T P\right)\right)=\sigma\left(B_{1}+T_{1}\right) \text {. }
$$

It is not hard to see that $B_{1}$ and $T_{1}$ satisfy $H_{1}$. For instance, since

$$
\left(\mu_{0} Q-B Q\right)(\nu-T)^{-1}=(\nu-T)^{-1}\left(\mu_{0} Q-B Q\right),
$$


it follows by Lemma 2 that $\mu_{0} Q-B Q: D(T) \rightarrow D(T)$ and $\left(\mu_{0} Q-B Q\right) T x=$ $T\left(\mu_{0} Q-B Q\right) x$ for $x \in D(T)$. Thus by Marti's theorem $\sigma\left(B_{1}+T_{1}\right) \subset \sigma\left(B_{1}\right)+\sigma\left(T_{1}\right)$. However, by the same arguments used in the first section, $\sigma\left(T_{1}\right) \subset N_{\varepsilon}($ ess $T) \cup V_{2 R}^{\prime}(0)$ and $\sigma\left(B_{1}\right) \subset N_{\varepsilon}($ ess $B)$. Putting all this together, $\lambda_{0} \in\left(N_{\varepsilon}(\right.$ ess $\left.T) \cup V_{2 R}^{\prime}(0)\right)+N_{\varepsilon}($ ess $B)$. However, it is impossible that $\lambda_{0}=\nu+\mu$ for $\nu \in V_{2 R}^{\prime}(0)$ and $\mu \in N_{\varepsilon}($ ess $B)$, since $\nu+\mu>2 R-R=R$ and $\left|\lambda_{0}\right| \leqq R$. Thus $\lambda_{0} \in N_{\varepsilon}($ ess $T)+N_{\varepsilon}($ ess $B)$. Since $\lambda_{0}$ was an arbitrary element of ess $(T+B)$, this shows that ess $(T+B) \subset N_{\varepsilon}$ (ess $\left.T\right)+N_{\varepsilon}$ (ess $\left.B\right)$. Q.E.D.

Notice that the above proof shows ess $(T+B)$ is empty if ess $(T)$ is empty.

Before we prove the corresponding theorem for ess $(B T)$, we need another lemma.

Lemma 5. Let $B$ and $T$ be linear operators satisfying $H_{2}$. Assume that $\sigma(T) \neq C$ and $0 \notin$ ess $(B)$. Then $B T$ is a closed linear operator. Furthermore, there exists a bounded, finite dimensional linear operator $F$ such that $B_{1}=B-F$ is invertible, $B_{1}$ and $T$ satisfy $H_{2}$, ess $\left(B_{1}\right)=$ ess $(B)$, and ess $\left(B_{1} T\right)=\operatorname{ess}(B T)$.

Proof. If $B$ is invertible, the lemma is immediate (even without the assumption that $\sigma(T) \neq C)$. Thus assume $0 \in \sigma(B)$-ess $(B)$. Let $C$ be a circle about 0 which contains no other points of $\sigma(B)$, and let $Q=(1 / 2 \pi i) \int_{C}(\mu-B)^{-1} d \mu$, a bounded, finite dimensional projection. We define $F=B Q-Q$, and we have to show $F$ satisfies the claims of the lemma.

The usual argument shows that $0 \notin \sigma(B-F)$, so that $B_{1}$ is invertible. It is easy to show that $(\nu-T)^{-1} F=F(\nu-T)^{-1}$ for $\nu \in \rho(T)$, so that by Lemma 2 we know that $F(X) \subset \bigcap_{j \geqq 1} D\left(T^{j}\right)$ and $(T F) x=(F T) x$ for $x \in D(T)$. It follows in particular that since $F(X)$ is a finite dimensional subspace of $D(T), T F$ is actually a bounded, finite dimensional linear operator. Since $B_{1}$ is invertible, $B_{1} T$ is closed and thus $B T=B_{1} T+T F$, a sum of a closed and a bounded linear operator, is closed.

To see that $B_{1}$ and $T$ satisfy $H_{2}$, it suffices to show $F$ and $T$ satisfy $H_{2}$, and we have already seen that $(F T) x=(T F) x$ for $x \in D(T)$. Trivially, $B F=F B$, so that by Lay's theorem, ess $\left(B_{1}\right)=$ ess $(B)$. Finally, to show that ess $\left(B_{1} T\right)=$ ess $(B T)$, it suffices, by Lay's theorem, to show that $(B T)(T F) x=(T F) x(B T) x$ for $x \in D(T)$. However, $F x \in \bigcap_{j \geqq 1} D\left(T^{j}\right)$ for any $x$, so that $(T F) x \in D(T)$, and we can write $(B T)(T F) x$ $=(T B)(T F) x$. If $x \in D(T)$, we also have $(T B)(T F) x=(T B)(F T) x=(T F)(B T) x$. Q.E.D.

Theorem 4. Let $B$ and $T$ satisfy $H_{2}$. Assume that $0 \notin$ ess $(B)$ and that $B T$ is closed (under the hypotheses, Lemma 5 implies that $B T$ is closed if $\sigma(T) \neq C$ ). Then ess $(B T) \subset \operatorname{ess}(B)$ ess $(T)=\{\nu \mu \mid \mu \in \operatorname{ess}(B), \nu \in$ ess $(T)\}$. If ess $(T)$ is empty, ess $(B)$ ess $(T)$ is interpreted as being empty.

Proof. Since ess $(B) \neq \varnothing$ and $0 \notin \operatorname{ess}(B)$ (recall that we assume $X$ infinite dimensional), we can select $\mu_{0} \in \operatorname{ess}(B), \mu_{0} \neq 0$. If $\sigma(T)=C$, then we have ess $(T)$ ess $(B) \supset\left\{\lambda \mu_{0} \mid \lambda \in C\right\} \supset C$, so that ess $(B T) \subset$ ess $(B)$ ess $(T)$. Thus we can 
assume that $\sigma(T) \neq C$. By Lemma 5 we can find a bounded, invertible operator $B_{1}$ such that $B_{1}$ and $T$ satisfy $H_{2}$, ess $\left(B_{1}\right)=\operatorname{ess}(B)$ and ess $\left(B_{1} T\right)=\operatorname{ess}(B T)$. Thus it suffices to show that ess $\left(B_{1} T\right) \subset \operatorname{ess}\left(B_{1}\right)$ ess $(T)$. For notational convenience, we shall write $B$ instead of $B_{1}$, but now we can also assume $B$ invertible.

Select $\lambda_{0} \in$ ess $(B T)$. Notice that to show $\lambda_{0} \in$ ess $(B)$ ess $(T)$, it suffices to show that $\lambda_{0} \in N_{\varepsilon}($ ess $B) N_{\varepsilon}($ ess $T)$ for all $\varepsilon>0$. For suppose $\lambda_{0} \in N_{\varepsilon}($ ess $B) N_{\varepsilon}($ ess $T)$ for all $\varepsilon>0$. Since ess $(B)$ is closed and $0 \notin$ ess $(B)$, we can assume that $|\mu| \geqq \delta>0$ for $\mu \in N_{\varepsilon}($ ess $B)$ and $0<\varepsilon<\varepsilon_{0}$. For $0<\varepsilon<\varepsilon_{0}$, it follows that if $\lambda_{0}=\mu \nu, \mu \in N_{\varepsilon}$ (ess $B$ ) and $\nu \in N_{\varepsilon}$ (ess $T$ ), then $|\nu| \leqq M=\left|\lambda_{0}\right| / \delta$. Thus (for $n$ large enough), if we select $\mu_{n} \in N_{1 / n}($ ess $B)$ and $\nu_{n} \in N_{1 / n}$ (ess $T$ ) such that $\mu_{n} \nu_{n}=\lambda_{0}, \mu_{n}$ and $\nu_{n}$ lie in compact sets, and by taking subsequences we can assume $\mu_{n} \rightarrow \mu \in$ ess $(B)$ and $\nu_{n} \rightarrow \nu$ $\in$ ess $(T)$. Thus we see that $\lambda=\mu \nu \in$ ess $(B)$ ess $(T)$.

We now proceed as in Theorem 3, though we shall omit many details. Take $\lambda_{0} \in$ ess $(B T)$. Select $\varepsilon>0$ small enough so that $|\mu| \geqq \delta>0$ for $\mu \in N_{\varepsilon}$ (ess $\left.B\right)$. Select a constant $R>\lambda_{0} / \delta$ and a number $\nu_{0}$ with $\left|\nu_{0}\right| \geqq R$. Select $\mu_{0} \in \operatorname{ess}(B)$ as before. Just as in Theorem 3, we can find finite dimensional projections $P$ and $Q$ which correspond respectively to the eigenvalues $\left\{\nu_{1}, \ldots, \nu_{n}\right\}=V_{R}(0) \cap\left(\sigma(T)-N_{\varepsilon}\right.$ ess $\left.(T)\right)$ and $\left\{\mu_{1}, \ldots, \mu_{m}\right\}=\sigma(B)-N_{\varepsilon}$ (ess $\left.B\right)$. We want to use Lay's theorem to show that

$$
\text { ess }(B T)=\text { ess }\left(\left(B+\mu_{0} Q-B Q\right)\left(T+\nu_{0} P-T P\right)\right) \text {. }
$$

We write $F_{1}=\mu_{0} Q-B Q$ and $F_{2}=\nu_{0} P-T P$. It is easy to show that $(\nu-T)^{-1} F_{1}$ $=F_{1}(\nu-T)^{-1}$ and $(\nu-T)^{-1} F_{2}=F_{2}(\nu-T)^{-1}$ for $\nu \in \rho(T)$. It follows by Lemma 2 that $F_{i}(X) \subset \bigcap_{j \geqq 1} D\left(T^{j}\right),\left(T F_{i}\right) x=\left(F_{i} T\right) x$ for $x \in D(T)$, and $T F_{i}$ is a bounded, finite dimensional linear operator, $i=1,2$. Using these results, we see that if we write $\left(B+F_{1}\right)\left(T+F_{2}\right)=B T+F, F$ is a bounded, finite dimensional linear operator and $(\nu-T)^{-1} F=F(\nu-T)^{-1}$ for $\nu \in \rho(T)$. Applying Lemma 2 again shows $F(X)$ $\subset D(T)$ and $(T F) x=(F T) x$ for $x \in D(T)$. Since it is obvious that $F B=B F$, Lay's theorem now implies ess $(B T+F)=\operatorname{ess}(B T)$.

Using the above results, we see that $B_{2}=B+F_{1}$ and $T_{2}=T+F_{2}$ satisfy the hypotheses of Lemma 4, so ess $\left(B_{2} T_{2}\right) \subset \sigma\left(B_{2} T_{2}\right) \subset \sigma\left(B_{2}\right) \sigma\left(T_{2}\right)$. Just as before, $\sigma\left(B_{2}\right)$ $\subset N_{\varepsilon}$ (ess $B$ ) (so, in particular, $B_{2}$ is invertible), and $\sigma\left(T_{2}\right) \subset N_{\varepsilon}$ (ess $\left.T\right) \cup V_{R}^{\prime}(0)$. By the selection of $R, \lambda_{0} \neq \mu \nu$ for $\mu \in N_{\varepsilon}$ (ess $\left.B\right)$ and $\nu \in V_{R}^{\prime}(0)$, so that $\lambda_{0} \in$ $N_{\varepsilon}$ (ess $\left.T\right) N_{\varepsilon}$ (ess $T$ ). As we have remarked, this establishes our theorem. Q.E.D.

Our next goal is to establish a perturbation theorem when $B$ may not be bounded. To compensate for the unboundedness of $B$, we have to strengthen our assumptions on $T$.

THEOREM 5. Let $T$ and $B$ be closed, densely defined linear operators with $D(T)$ $\subset D(B)$. Let $D=\{x \in D(T) \mid T x \in D(B)\}$ and assume $B: D \rightarrow D(T)$ and $(T B) x$ $=(B T) x$ for $x \in D$. Assume that $T+B$ is closed and $\rho(T+B)$ is nonempty. Finally, suppose ess $(T)$ is compact. Then there exists a closed subspace $X_{1}$ of $X$ and a bounded linear operator $B_{1}: X_{1} \rightarrow X_{1}$ such that ess $\left(B_{1}\right) \subset \operatorname{ess}(B)$ and ess $(T+B) \subset \operatorname{ess}(T)$ + ess $\left(B_{1}\right)$. 
Proof. For $\nu \in \rho(T)$ and $x \in D(B)$, we have $B(\nu-T)^{-1} x=(\nu-T)^{-1} B x$, because $(\nu-T)^{-1} x \in D$ for $x \in D(B)$ and then $(\nu-T) B(\nu-T)^{-1} x=B(\nu-T)(\nu-T)^{-1} x$ $=(\nu-T)(\nu-T)^{-1} B x$. Since ess $(T)$ is compact, let $C$ be a simple Jordan curve enclosing ess $(T)$ and lying in $\rho(T)$. Define $P=(1 / 2 \pi i) \int_{C}(\nu-T)^{-1} d \nu$ and set $X_{1}=P X, X_{2}=(I-P) X$. As usual, $P: X \rightarrow D(T)$, and in fact since $P$ is a projection, $P: X \rightarrow \bigcap_{j \geqq 1} D\left(T^{j}\right)$. Since $(\nu-T)^{-1} B x=B(\nu-T)^{-1} x$ for $x \in D(B)$, we find easily that $(B P) x=(P B) x$ for $x \in D(B)$. It follows that for $u \in X_{1}, B u=B P^{2} u=P B P u \in X_{1}$. Thus we can define $B_{1}: X_{1} \rightarrow X_{1}$ by $B_{1} u=B u, u \in X_{1}$. Similarly, we define $T_{1}: X_{1} \rightarrow X_{1}$. Since both $B_{1}$ and $T_{1}$ are closed and defined on all of $X_{1}$, they are bounded. Similarly, we note that $B: X_{2} \cap D(B) \rightarrow X_{2}$ and $T: X_{2} \cap D(T) \rightarrow X_{2}$, so we define $B_{2}=B \mid X_{2} \cap D(B)$ (viewed as a map into $X_{2}$ ) and $T_{2}=T \mid X_{2} \cap D(T)$ (viewed as a map into $X_{2}$ ).

Standard results now imply that $\sigma(B)=\sigma\left(B_{1}\right) \cup \sigma\left(B_{2}\right), \sigma(T)=\sigma\left(T_{1}\right) \cup \sigma\left(T_{2}\right)$ and $\sigma(T+B)=\sigma\left(T_{1}+B_{1}\right) \cup \sigma\left(T_{2}+B_{2}\right)$. Using these results and Browder's original definition of the essential spectrum, it is not hard to see that ess $(B)=\operatorname{ess}\left(B_{1}\right)$ $\cup$ ess $\left(B_{2}\right)$, ess $(T)=\operatorname{ess}\left(T_{1}\right) \cup \operatorname{ess}\left(T_{2}\right)$ and ess $(T+B)=\operatorname{ess}\left(T_{1}+B_{1}\right) \cup \operatorname{ess}\left(T_{2}+B_{2}\right)$. We omit the proof. However, we know as usual that $\sigma\left(T_{2}\right)$ lies outside $C$, so that ess $\left(T_{2}\right)$ lies outside $C$. Since $C$ encloses ess $(T)$, ess $\left(T_{1}\right)=\operatorname{ess}(T)$ and ess $\left(T_{2}\right)$ is empty. Note also that $B_{1} T_{1}=T_{1} B_{1}$. To show this, it suffices to show that $(B T)(P x)$ $=(T B)(P x)$ for $x \in X$, and this is true since $P x \in D\left(T^{2}\right) \subset D$. It follows by Theorem 3 that ess $\left(T_{1}+B_{1}\right) \subset$ ess $\left(T_{1}\right)+$ ess $\left(B_{1}\right)=$ ess $(T)+$ ess $\left(B_{1}\right)$. Thus to complete the proof it suffices to show that ess $\left(T_{2}+B_{2}\right)$ is empty.

Since we assume $\rho(T+B)$ is nonempty, take $\mu_{0} \in \rho(T+B)$, so that certainly $\mu_{0} \in \rho\left(T_{2}+B_{2}\right)$. Similarly, take $\nu_{0} \in \rho(T)$, so that $\nu_{0} \in \rho\left(T_{2}\right)$. Then we can write

$$
\text { ess } \begin{aligned}
\left(T_{2}+B_{2}\right) & =\mu_{0}+\operatorname{ess}\left(-\mu_{0}+T_{2}+B_{2}\right) \\
& =\operatorname{ess}\left(\left(-\mu_{0}+T_{2}+B_{2}\right)\left(\nu_{0}-T_{2}\right)^{-1}\left(\nu_{0}-T_{2}\right)\right)+\mu_{0} .
\end{aligned}
$$

If we set $B_{3}=\left(-\mu_{0}+T_{2}+B_{2}\right)\left(\nu_{0}-T_{2}\right)^{-1}, B_{3}$ is a bounded, invertible operator. It is also easy to check that $B_{3}: D\left(T_{2}\right) \rightarrow D\left(T_{2}\right)$ and $B_{3}\left(\nu_{0}-T_{2}\right) x=\left(\nu_{0}-T_{2}\right) B_{3} x$ for $x \in D\left(T_{2}\right)$. It follows by Theorem 4 that ess $\left(B_{3}\left(\nu_{0}-T_{2}\right)\right) \subset$ ess $\left(B_{3}\right)$ ess $\left(\nu_{0}-T_{2}\right)$. However, ess $\left(\nu_{0}-T_{2}\right)=\nu_{0}-\operatorname{ess}\left(T_{2}\right)$ is empty, so that ess $\left(B_{3}\left(\nu_{0}-T_{2}\right)\right)$ is empty and ess $\left(T_{2}+B_{2}\right)$ is empty. Q.E.D.

Corollary 1. Let $T$ and $B$ be closed, densely defined linear operators with $D(T) \subset D(B)$. If $D=\{x \in D(T) \mid T x \in D(B)\}$, assume that $B: D \rightarrow D(T)$ and $(T B) x=(B T) x$ for $x \in D$. Assume that ess $(T)$ is compact. Suppose that there exists a sequence $\lambda_{n} \in \rho(T),\left|\lambda_{n}\right| \rightarrow \infty$, such that $\left\|\left(\lambda_{n}-T\right)^{-1}\right\| \leqq C /\left|\lambda_{n}\right|, C$ a constant. Finally, assume that for every $\varepsilon>0$, there is a constant $K_{\varepsilon}$ such that $\|B x\| \leqq \varepsilon\|T x\|$ $+K_{\varepsilon}\|x\|$ for $x \in D(T)$. Then the conclusion of Theorem 5 holds.

Proof. It is known (see [4]) that if $\|B x\| \leqq c\|T x\|+K\|x\|$ for $x \in D(T)$ with $c<1$, then $T+B$ is closed. Thus $T+B$ is certainly closed. To apply Theorem 5 , it only remains to show that $\rho(T+B)$ is nonempty. We claim that $\lambda_{n} \in \rho(T+B)$ for $n$ 
large enough, and since $\left(\lambda_{n}-T-B\right)=\left(I-B\left(\lambda_{n}-T\right)^{-1}\right)\left(\lambda_{n}-T\right)$, it suffices to show that $\left\|B\left(\lambda_{n}-T\right)^{-1}\right\|<1$ for $n$ large enough. But by our hypothesis,

$$
\begin{aligned}
\left\|B\left(\lambda_{n}-T\right)^{-1} x\right\| & \leqq \varepsilon\left\|T\left(\lambda_{n}-T\right)^{-1} x\right\|+K_{\varepsilon}\left\|\left(\lambda_{n}-T\right)^{-1} x\right\| \\
& =\varepsilon\left\|\lambda_{n}\left(\lambda_{n}-T\right)^{-1} x-x\right\|+K_{\varepsilon}\left\|\left(\lambda_{n}-T\right)^{-1} x\right\| \\
& \leqq \varepsilon\|x\|+\varepsilon C\|x\|+K_{\varepsilon} /\left|\lambda_{n}\right|\|x\| .
\end{aligned}
$$

Thus we merely select $\varepsilon$ such that $\varepsilon+\varepsilon C<1$ and then select $n$ so large that

$$
\varepsilon+\varepsilon C+K_{\varepsilon} /\left|\lambda_{n}\right|<1 \text {. }
$$

\section{REFERENCES}

1. F. E. Browder, On the spectral theory of elliptic differential operators. I, Math. Ann. 142 (1960/61), 22-130. MR 35 \#804.

2. I. C. Gohberg and M. G. Kreĭn, The basic propositions on defect numbers, root numbers and indices of linear operators, Uspehi Mat. Nauk 12 (1957), no. 2 (74), 43-118; English transl., Amer. Math. Soc. Transl. (2) 13 (1960), 185-264. MR 22 \#3984.

3. S. Goldberg, Unbounded linear operators: Theory and applications, McGraw-Hill, New York, 1966. MR 34 \#580.

4. T. Kato, Perturbation theory for linear operators, Die Grundlehren der math. Wissenschaften, Band 132, Springer-Verlag, Berlin and New York, 1966. MR 34 \#3324.

5. D. Lay, Characterizations of the essential spectrum of F. E. Browder, Bull. Amer. Math. Soc. 74 (1968), 246-248. MR 36 \#4370.

6. J. T. Marti, Operational calculus for two commuting closed operators, Comment. Math. Helv. 43 (1968), 87-97. MR 36 \#6967.

7. A. E. Taylor, Theorems on ascent, descent, nullity, and defect of linear operators, Math. Ann. 163 (1966), 18-49. MR 32 \#8169.

8. T. T. West, Riesz operators in Banach spaces, Proc. London Math. Soc. (3) 16 (1966), 131-140. MR 33 \#1742.

RUTGERS UNIVERSITY,

New Brunswick, NeW JerSey 08903 